\title{
Investigating insight and rigour as separate constructs in mathematical proof
}

\author{
Chris Sangwin* and George Kinnear ${ }^{\dagger}$
}

3rd June 2021

\begin{abstract}
In this paper we investigate undergraduate mathematics students' conceptions of rigour and insight. We conducted comparative judgement experiments in which students were asked to judge different proofs of the same theorem with five separate criteria: rigour, insight, understanding, simplicity and assessment marks. We predicted, and our experiment found, that rigour is a reliable construct. We predicted that insight is also a reliable construct but asking students to judge on the basis of "which proof gives you more insight into why a theorem is true" did not result in a reliable judging scale. Our analysis suggests two distinct dimensions: rigour and marks contribute to one factor whereas simplicity and personal understanding relate to a second factor. We suggest three reasons why insight was related almost equally to both factors. First, while comparative judgement was suitable for assessing some aesthetic criteria it may not be suited to investigating students conceptions of insight. Second, students may not have developed an aesthetic sense in which they appreciate insight in ways which are regularly discussed by mathematics educators. Lastly, insight may not be a coherent and well-defined construct after all.
\end{abstract}

Keywords: insight and rigour; mathematical proof; comparative judgement; aesthetics.

\section{Introduction}

Proof is a central feature of mathematics, indeed proof is the hallmark which distinguishes mathematics from empirical science or philosophical debate. Mathematicians and philosophers of mathematics routinely make distinctions between proofs on aesthetic terms: "Intuitively, some proofs establish that a theorem holds, while others show why it holds. Proofs of the latter kind are often called explanatory." (Hamami \& Morris, 2020, p. 1119). While intuitive, this distinction has been the subject of much debate.

On the one hand, proving that a theorem holds is independent of human interpretation: it is a matter of providing a rigorous proof. A commonly-used definition is that "a mathematical proof is rigorous whenever it can be routinely translated into a formal proof (Mac Lane 1986, p. 377)" (Hamami \& Morris, 2020, p. 1117). Formalization has a long and important history going back through Russell to Leibnitz, who sought for a universal scientific language by which mathematicians could "judge immediately whether propositions presented to us are proved ... with the guidance of symbols alone, by a sure truly analytical method." (cited by MacKenzie, 2004, p. 15). Indeed, we are at a time in which professional mathematicians are gradually making more use of formal proof checkers, with a growth of formalization of proofs within mathematical research (Avigad, 2018). This change is gradual, ongoing, and by no means a universally accepted process (see e.g., MacKenzie, 2004).

On the other hand, for a proof to show why a theorem holds, human understanding is a crucial concern:

\footnotetext{
*School of Mathematics, University of Edinburgh, Edinburgh, EH9 3FD. C. J . Sangwin@ed . ac . uk

${ }^{\dagger}$ School of Mathematics, University of Edinburgh, Edinburgh, EH9 3FD. G. Kinnear@ed. ac. uk
} 
We want to understand the proof, not just be told it exists. What the proof should do for the student is provide insight into why the theorem is true. (Hersh, 1993)

Based on interviews with research-active mathematicians, Weber (2010, p. 36) noted two further forms of insight that can be gained from proofs: "methods that might be useful for the problems they were researching" and "ways to reconceive the domains they were studying". The latter sense is related to what Bell (1976) termed systematisation, i.e. "the organisation of results into a deductive system of axioms, major concepts and theorems, and minor results derived from these." These different forms of insight could all be seen to fit with the view of Inglis and Mejía-Ramos (2019) that "a proof is explanatory for a given reader if it brings about understanding for that reader"-while Inglis and Mejía-Ramos focus on only the first type of insight as their definition of explanatory ("While all proofs establish their theorems, apparently some go further and explain why the theorem holds true."), their argument about "maximal understanding" and schema-building should apply equally well to the other types of insight identified by Weber (2010).

These two properties of proofs-rigour and insight—are educationally relevant, with mathematics educators typically arguing for the importance of explanatory or insightful proofs: "The role of proof in the mathematics classroom is primarily explanatory; that is, students should ideally view proofs as giving insight into why propositions are true or false." (Smith, 2006, p. 75); see also (Hanna, 1990; Hersh, 1993). Stylianides et al. (2016, p. 23) observed that explanatory power "can help students develop their understanding of the mathematical ideas involved (ideas of number theory in our study), ideas about proof, or both.", which is related to Freudenthal's "fourth major problem in mathematics education":

How to keep open the sources of insight during the training process, how to stimulate retention of insight, in particular in the process of schematising? (Freudenthal, 1981, pp. 140-141)

Rigour and insight can sometimes be seen as being in direct tension, as in Brown's (2008) discussion of the merits of visual proofs:

I would be seriously misunderstood, if it were thought that I am suggesting it is worth giving up some rigour in exchange for insight. This is doubly wrong. I don't see any abandoning of rigour by allowing the legitimacy of picture-proofs. And second, greater insight isn't always to be found in pictures. (Brown, 2008, p. 46)

Others have acknowledged practical difficulties in selecting proofs which are likely to help students most:

When choosing which proof of a theorem to present and how to present it, an author or teacher would be helped by having explicit criteria for the degree to which a proof is explanatory and fosters understanding. Unfortunately there are no such well-defined and shared criteria. (Hanna \& Mason, 2016)

There is some recent empirical evidence to support distinguishing proofs on the basis of rigour and insight. Inglis and Aberdein (2015) asked mathematicians to rate the extent to which 80 different adjectives applied to a proof they had read recently, and used factor analysis to identify 5 groups of adjectives where the ratings were correlated. The adjective insightful appeared in a group termed 'Aesthetics' (alongside words such as ingenious, striking and inspired), while rigorous appeared in a different group, termed 'Precision' (along with words like careful, meticulous and precise). In subsequent work, Inglis and Aberdein (2016) used a subset of 20 adjectives to gather mathematicians' opinions about a particular proof, and found that there was a diverse range of opinions. They argued that this evidence of a lack of consensus on the properties of a proof presents a serious problem for the approach of those they term "exemplar philosophers", who "have typically relied upon their own intuitions about the qualities of a proof to draw philosophical conclusions" (p. 174). This sentiment is in accord with the empirical approach we take in this paper to investigate whether the properties of insight and rigour can be robustly distinguished. 
In more recent work, Mejía-Ramos et al. (2021) investigated mathematicians' views of the explanatory value of nine proofs, taken from a collection of different proofs of the same result (Ording, 2019). They used a comparative judgement approach, where the mathematicians made a series of paired comparisons between proofs, each time choosing the one they thought "best explains why the proposition holds". Analysis of the set of judgements showed a high level of agreement between the mathematicians, demonstrating the success of the comparative judgement approach in measuring aesthetic perceptions.

\subsection{Research focus}

While the distinction between rigour and insight is widely discussed in educational and philosophical literature, can we find any evidence that mathematicians evaluate the quality of proofs differently when prompted to distinguish on the grounds of rigour or insight? That is to say, when asked to choose between two proofs on the basis of which has more "rigour"/"insight" do mathematicians agree? Are these constructs different, or are they indistinguishable?

In this paper, we build on the approach of Mejía-Ramos et al. (2021), by using comparative judgement to gather undergraduate mathematics students' opinions on the rigour and insight of a set of proofs of the same theorem. Our main contribution is to extend the approach to investigate potential differences between two constructs-rigour and insight. In the next section, we give an overview of the comparative judgement method, and discuss the collection of proofs that we selected for the studies, in particular we address concerns we have about the use of the proofs from (Ording, 2019). Following that, we report on two studies carried out using this method.

\section{Method}

\subsection{Comparative judgement}

In this paper we use the method of comparative judgement (CJ). The practical CJ process is very simple: judges are presented with pairs of items and asked to decide which is "better" in terms of some explicit construct such as "more rigourous" or "provides better insight". The underlying theoretical basis is a wellestablished psychological principle that people are more reliable when comparing one sense impression against another than they are at judging an impression in isolation (Thurstone, 1927). Judges must make a choice and cannot choose a 'tied' result. The judges' decisions are then used, with a statistical model, to produce a standardised parameter estimate (z-score) for each item being judged, together with a standard error giving some indication of the uncertainty of the estimate.

The reliability of the scores produced by $\mathrm{CJ}$ can be measured in two ways: the scale separation reliability (SSR) which comes from the underlying statistical model (Bramley, 2015), and the split-halves inter-rater reliability (Bisson et al., 2016), which comes from repeatedly splitting the judges into two subgroups, computing the correlation between the scores produced by each group, and taking the mean over all the iterations. Both measures produce a number between 0 and 1 , with 1 representing perfect reliability, and 0.7 commonly used as a threshold of acceptable reliability. There is some indication that the two measures are related, with the split-halves measure being systematically lower than SSR (Verhavert et al., 2018).

CJ is a well-established technique for assessing students' work (Jones et al., 2014; Pollitt, 2012), by using the parameter to allocate grades to students. Judges could be experts, or CJ can be used for peerassessment (e.g., Jones \& Alcock, 2014). A variety of online tools are available to facilitate the process of orchestrating and presenting items to judges (e.g., Jones, 2021).

$\mathrm{CJ}$ is also becoming a well-established as a research tool (e.g., Bisson et al., 2016; Jones et al., 2019). As noted above, Mejía-Ramos et al. (2021) used CJ to determine mathematicians' views of the explanatory value of a collection of proofs. Other recent work has used CJ to investigate students' comprehension of proofs by judging the quality of their written summaries of proofs (Davies et al., 2020), and to investigate 
mathematicians' and undergraduate students written conceptions of mathematical proof (Davies et al., 2021).

\subsection{Collection of proofs}

To control for possible differences resulting from the choice of theorem, we set out to find a collection of many proofs of the same theorem. There is a long tradition of mathematicians valuing collections of proofs. For instance, Loomis (1968) collected proofs of the Pythagorean Theorem, and Kalman (1993) gathered six different ways to prove that $\sum_{n=1}^{\infty} \frac{1}{n^{2}}=\frac{\pi^{2}}{6}$. The recent work of Ording (2019) is an inspirational discussion of mathematical style via a comparison of 99 different "proofs" of the theorem

$$
\text { Let } x \in \mathbb{R} \text {. If } x^{3}-6 x^{2}+11 x-6=2 x-2 \text { then } x=1 \text { or } x=4 \text {. }
$$

The theorem chosen by Ording (2019) is consciously elementary, but as a vehicle to investigate students' conceptions of proof the collection has, in our view, a number of serious shortcomings. Most notably, for our participants the mathematics is too elementary: so elementary that one might question whether the result deserves the adjective "theorem" beyond a very formal sense.

Instead, to investigate the research questions we sought to identify a more suitable theorem with a collection of proofs in different mainstream styles. Ultimately we chose the following.

Theorem 1 The sum of the first $n$ odd integers, starting from one, is $n^{2}$.

In algebraic notation, this theorem is $1+3+5+\cdots+(2 n-1)=\sum_{k=1}^{n}(2 k-1)=n^{2}$. There are several reasons why we opted for this theorem. First, Ording's theorem is specific to his cubic and is not a general theorem in the sense defined by Sangwin and Bickerton (2021). That is, a "specific theorem" pertains to a single situation (e.g. $\sqrt{2}$ is irrational) whereas a "general theorem" proves a common property of a class of examples, possibly collected via the device of a formal definition. Since the equation in Theorem 1 holds for all natural numbers $n$ we consider Theorem 1 to be a general theorem.

Second, in addition to being general, Theorem 1 is representative of a wide class of similar results. That is, ideas and techniques in many of the proofs can be readily recycled in a wider range of situationswhat Raman-Sundström and Öhman (2016) describe as "generality". In particular, Theorem 1 is related to finding formulae for $\sum_{k=1}^{n} k^{m}$, for $m=0,1,2,3,4, \ldots$. For example, $m=1$ gives $1+2+3+\cdots+n=\frac{n(n+1)}{2}$. Sums of powers of integers have an important history (Burn, 2010; Edwards, 1982).

Third, Theorem 1 has a level of technical complexity in the statement of the theorem, and the proofs, which in our judgement as teachers (and examiners) is relatively simple for year 1 undergraduate students. The topic of finite series is very familiar for our participants (undergraduate students at a leading UK university). By way of contrast, geometry would also provide many suitable theorems for this study, but was rejected because geometry is not well studied in the UK system.

Fourth, we argue that Theorem 1 is a result which typical students might agree "needs to be proved". That is to say, a theorem which is sufficiently complex that a typical reader would not immediately see why the theorem is true, and yet is not so foundational that the theorem is itself a technical demonstration of rigour. For example, as late as 1872 Dedekind remarked that $\sqrt{2} \sqrt{3}=\sqrt{6}$ had not been proved rigorously, (Dedekind, 1963, p. 22), and our students might also not appreciate why such a proof was needed either. These are both intellectual needs in the first sense discussed by (Harel, 2014) as the need for certainty.

Fifth, we wanted a theorem for which a wide selection of different proof styles exist, including direct proof, induction, and contradiction. More particularly we wanted proofs which either have, or might be, presented to students as possessing some genuine merit: rigorous, insightful, elegant, representative of a proof style, or the criteria proposed by Raman-Sundström and Öhman (2016). This is in direct contrast to the rather special arguments given by Aigner and Ziegler (2018) in their widely respected collection of proofs, some of which have been used for educational research (e.g., Inglis \& Aberdein, 2016). By their very nature these 
are atypical mathematical arguments: that is precisely why they have been collected together. Similarly, some of the proofs in (Ording, 2019) are outlandish and are unlikely to ever arise "naturally" in a discussion with students. There are many ways to prove Theorem 1: in preparation for this research we considered over 20 different proofs of the result (see Sangwin, 2023). Indeed, it turns out that we are not the first to suggest students use Theorem 1 to collect different proof techniques as an exercise.

3.12 Find $1+3+5+\cdots+(2 n-1)$, the sum of the first $n$ odd numbers. (List as many different approaches as you can.). (Polya, 1962, I. pg. 81)

Ultimately 13 distinct proofs were selected as appropriate for the participant group, and the variants used in our studies are reproduced in Appendix A.

\section{Study 1}

We conducted a comparative judgement experiment asking students to judge proofs of Theorem 1 on the basis of "insight" or "rigour". We aimed to address the questions:

1. Do students distinguish insight and rigour?

2. What are features of proofs that students score high/low on each dimension?

Our expectation, based on the literature discussed in section 1, was that results would be consistent with theory suggesting that rigour and insight are each coherent constructs. Moreover, we expected that there would be substantial differences between the scores on each dimension. Specifically, we anticipated that pictorial proofs and experimental evidence will score very low on rigour but will be judged to provide insight. We also expected problem solving proofs, where the result is derived in the course of the proof rather than assumed a priori, to be judged as providing more insight. Specifically, we judged 4 (arithmetic progression), 5 (reversed lists), 7 (backwards reasoning), 8 \& 9, (rearranging I \& II), 12 (linear system) and 13 (undetermined coefficients) to be problem solving proofs.

\subsection{Method}

Participants were enrolled on a course Honours Complex Variables in year 3 of a mathematics degree. Students were invited to read through the proofs included in the study as preparation for a workshop on mathematical writing, and to read the detailed instructions about the study, given in Appendix B.1. Students were then asked to choose whether or not to take part in the research study, and those students who agreed were randomly assigned to two groups, "insight" and "rigour". Before judging, students in each group were asked to write about their interpretation of the relevant judging criterion, as shown in the "Definition prompt' column in Table 1. This was intended to prompt participants to focus their attention on the assigned criterion, and also to give us a way of checking whether the judges interpreted the prompts as we intended.

Judging was then conducted with the Moodle "Assignment" module, using the optional comparative judgement submission plugin (Jones, 2021). Participants were presented with two proofs side-by-side and were asked to click "left" or "right" based on the relevant judging prompt shown in Table 1. Participants had the option to include a comment on why they made their selection. Participants were repeatedly presented with another two proofs randomly selected from the set of possible pairs. Participants were asked to complete a minimum of 10 judgements and were restricted to a maximum of 25 judgements.

All students, including those who did not consent to the study, were later provided with all the proofs in a single document to read and discuss at a subsequent course workshop. In total, 55 students participated in judging. The median time taken for each judgement was 14 seconds. To ensure the results are not skewed by participants not taking the task seriously, we removed all judgements from judges whose median 


\begin{tabular}{lll}
\hline Condition & $\begin{array}{l}\text { Definition prompt } \\
\text { Please explain briefly in a few sentences } \\
\text { how you would decide if a proof... }\end{array}$ & $\begin{array}{l}\text { Judging prompt } \\
\text { In each case select the proof which... }\end{array}$ \\
\hline rigour & is rigorous. & is more rigorous. \\
insight & gives you insight. & gives more insight. \\
\hline
\end{tabular}

Table 1: Prompts used in Study 1

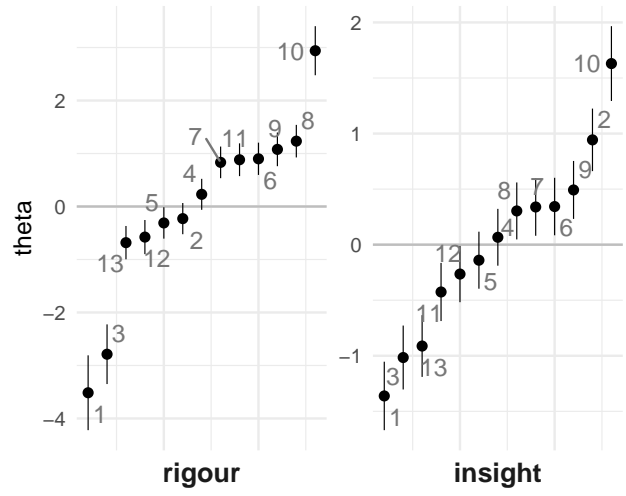

1. Experimental evidence

2. Pictorial I (L-shaped)

3. Pictorial II (Pyramids)
4. Arithmetic progression

5. Reversed list

6. Telescope

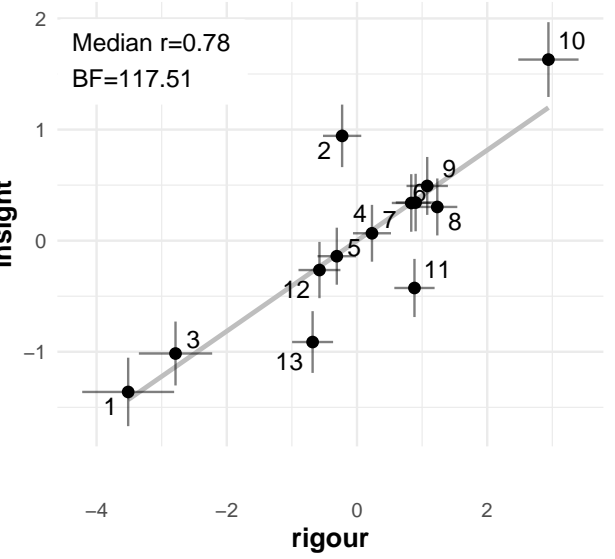

7. Backwards reasoning

8. Rearranging I

9. Rearranging II
10. Induction

11. Contradiction

12. Linear system

Figure 1: CJ scale scores, with standard errors, for insight and rigour in Study 1. On the left, each scale is shown separately. On the right, they are plotted against each other, along with the results of a Bayesian correlation test.

judgement time was under 5 seconds. This left 53 judges ( 24 for rigour and 29 for insight). Both judging sessions achieved an acceptable level of reliability as measured by the SSR, with 0.948 for rigour and 0.889 for insight. Split-halves reliability was also high ( 0.899 for rigour, 0.716 for insight) ${ }^{1}$

\subsection{Results}

For each judging session, the Bradley-Terry model produces an estimated position for each proof on a (unitless) scale, along with a standard error indicating the precision with which that position has been estimated. The position of each proof on the two dimensions is shown in Figure 1, and full results are recorded in Appendix C. Note that the scores for rigour and insight are on different scales (shown in the left half of Figure 1). Thus, it is not meaningful to directly compare the scores for a given proof on the two scales, but we can meaningfully consider the correlation between the two scales (much as if we were instead analysing the height and weight of 13 people).

\footnotetext{
${ }^{1}$ The number of judges is different in each case, so we also checked the split-halves reliability when choosing an equal number of judges in each case (similar to Mejía-Ramos et al., 2021). This gave similar results: 0.890 for rigour and 0.665 for insight.
} 


\subsubsection{Do students distinguish insight and rigour?}

The scores for rigour and insight are closely aligned, as can be see in the right half of Figure 1. A Bayesian correlation test (using a uniform prior) shows there is strong evidence in favour of a correlation between the rigour and insight scores, with a Bayes factor of $B F_{10}=117.51$. The median estimated correlation coefficient was 0.78 (95\% HDI [0.5, 0.94]). This suggests that, for this group of students, the two dimensions are closely aligned.

There are three outlier proofs for which the judges indicated a difference between rigour and insight. As predicted, 2 (pictorial I) scored low on rigour, but much higher on insight. However, 3 (pictorial II) scored very low in both: pictures alone do not necessarily give insight. Both 11 (contradiction) and 13 (undetermined coefficients) are outliers in the opposite direction, being scored higher in rigour than insight.

\subsubsection{What are features of proofs that students score high/low on each dimension?}

Looking at the results for rigour, 10 (induction) is clearly judged most rigorous by the students. Next are two groups of proofs which are harder to separate. Proofs 6 (telescope), 7 (backwards), $8 \& 9$ (rearranging I \& II), and 11 (contradiction) form one group of proofs judged as rigorous. Proofs 2 (pictorial I), 4 (AP), 5 (reversed list), 12 (linear system), 13 (undetermined coeffs) form another group, which are less rigorous. Two proofs, 3 (pictorial II) then 1 (experimental evidence), are clearly separated and were judged as least rigorous.

In our original research questions we noted interest in whether characteristics such as generality or problemsolving are factors in "rigour" and "insight". Given our finding there is surprisingly little difference between the results for insight and rigour we postpone discussion of these characteristics.

\subsection{Discussion}

We set out to determine whether the conceptions of "insight" and "rigour" of mathematical proofs can be distinguished by undergraduate mathematics students, with separate groups using a comparative judgement approach to evaluate a series of proofs using these criteria. The results showed that students' ratings of the proofs using the two criteria were strongly correlated (median estimated $r=0.78$ ), and only proofs 2 (pictorial I), 11 (contradiction) and 13 (undetermined coefficients) were outliers. This suggests that, at least for this group of students, "insight" and "rigour" have essentially the same meaning. This result is a surprise- it is in direct contradiction to our expectation, and predicted results.

A possible explanation comes from the responses that students gave before judging, to the prompt "please explain briefly in a few sentences how you would decide if a proof (is rigorous/gives you insight)". There was surprisingly little qualitative difference in the responses between the two groups. For example, the following is a typical response from the insight group:

A proof would give me insight if it is clear and concise. All the terms and processes should be well defined. Each step should be shown with no gaps in the proof.

After replacing "give me insight" with "be considered rigorous", such a response would not have been out of place from the rigour group. The comments confirmed the lack of quantitative difference in the rankings.

The similarity between the scores for insight and rigour could be explained in two ways. It may be that, for this group, insight does not exist as a construct which is separate from rigour. Alternatively, it could be that our method is flawed —and, in particular, we have some evidence that we have not prompted judges in a way which communicates the criteria which would reveal the difference.

We decided to conduct a second study to investigate the issue further and address possible concerns about the method. Further discussion is therefore postponed until after Study 2. 


\section{Study 2}

Our overarching aim is still to address the question of whether undergraduate students can distinguish between rigour and insight as properties of mathematical proofs. However, informed by the results of Study 1, we sought to address four more specific questions.

1. Do the results from Study 1 replicate? In particular, does a different group of undergraduate students engaged in comparative judgement produce similar scales for rigour and insight as in Study 1 ?

2. Can we adapt the judging instructions to better prompt students to use the intended meaning of "insight"?

3. Are students merely judging the proofs on some general criterion (e.g. "best")?

4. Does adapting certain proofs lead to predictable changes in their scores? In particular, for picture proofs, adding words of explanation should boost rigour, while for the induction proof, making the style less formal should reduce rigour.

\subsection{Method}

We used substantially the same method as in Study 1 to address these questions, with adaptations as outlined in the following sections.

\subsubsection{Procedure}

The overall procedure was similar, with participants randomly allocated to different judging groups. Before undertaking paired comparisons, the judges were asked to explain their interpretation of the judging criterion. We amended the stem of the prompt used in Study 1, replacing "how you would decide if a proof is ..." with "what it means for a proof to...", to encourage the judges to think more abstractly about the given property.

To address question 2 (investigating insight), we made two changes. First, we amended the wording of the prompts in the insight condition to specify "insight into why the theorem is true", echoing wording that is commonly used (e.g., Bell, 1976; Hersh, 1993; Smith, 2006), and attempting to direct attention away from other possible kinds of insight, such as insight into proof techniques or mathematical concepts (Weber, 2010). Second, we created an additional judging group, understanding, with a focus on whether the proof helps to develop personal understanding. For this group, the judging criterion is more explicitly related to epistemic accounts of explanatoriness, which "describe a proof as having explanatory value if and only if it grants some agent an understanding of why the associated theorem is true" (Inglis \& Mejía-Ramos, 2019, p. 7).

To address question 3 (independence of judging criteria), we sought evidence of divergent validity in the judges' scores by adding two further judging groups. First, the simple condition, which was chosen based on analysis of data from Inglis and Aberdein (2016) suggesting that simplicity was least likely to correlate with insight and rigour. Second, the marks condition, which was inspired by Healy and Hoyles (2000), who asked students to select the proof to which they judged a teacher would give most marks.

These changes resulted in five groups with different prompts for a definition (e.g. "what it means for a proof to be rigorous") and for judging (e.g. "which proof is more rigorous?"), as shown in Table 2.

\subsubsection{Materials}

To address question 4 (modified proofs), we used the same set of proofs as in Study 1, with two additions. First, we added a variant of the proof by induction that was deliberately less formal in style. The reason 


\begin{tabular}{|c|c|c|}
\hline Condition & $\begin{array}{l}\text { Definition prompt } \\
\text { Please explain briefly in a few sentences } \\
\text { what it means for a proof to... }\end{array}$ & $\begin{array}{l}\text { Judging prompt } \\
\text { Which proof... }\end{array}$ \\
\hline rigour & be rigorous & is more rigorous? \\
\hline insight & give insight into why the theorem is true & $\begin{array}{l}\text { gives more insight into why the theorem } \\
\text { is true? }\end{array}$ \\
\hline simple & be simple & is the simplest? \\
\hline understanding & $\begin{array}{l}\text { help you understand why a theorem is } \\
\text { true }\end{array}$ & $\begin{array}{l}\text { best helps you understand why the } \\
\text { theorem is true? }\end{array}$ \\
\hline marks & be worth high marks in an assessment & $\begin{array}{l}\text { would get the most marks in an } \\
\text { assessment? }\end{array}$ \\
\hline
\end{tabular}

Table 2: Prompts used in Study 2

\begin{tabular}{lrrrr}
\hline Dimension & Judges & Judgements & SSR & Split-halves reliability \\
\hline rigour & 39 & 636 & 0.942 & 0.880 \\
insight & 25 & 438 & 0.724 & 0.465 \\
simple & 32 & 526 & 0.911 & 0.822 \\
understanding & 34 & 611 & 0.845 & 0.659 \\
marks & 23 & 382 & 0.922 & 0.853 \\
\hline
\end{tabular}

Table 3: Details of the judging sessions in Study 2.

for this was to test whether proof by induction is inherently judged as rigorous, or whether (and how) the precise way a particular proof is written affects students' judgements. This is related to the "language as a fixed effect" concern in psychology (Clark, 1973). Second, we added a variant of 3 (pictorial II) that includes accompanying text to explain the reasoning. Varying 3 (pictorial II) was motivated by previous work finding that a visual proof was judged as "more persuasive when it was accompanied by a passage of descriptive text" (Inglis \& Mejía-Ramos, 2009). These additions are numbered 14 (induction b) and 15 (pictorial IIb) in Appendix A.

\subsubsection{Participants}

Participants were enrolled on a course Proofs and Problem Solving in year 1 of an undergraduate degree. The majority of students are taking a mathematics degree, although the course has students from other degrees, notably computer science. Students were invited to read through the proofs during week 7 of the semester as preparation for discussion of proofs later in the course. Participation in the study was voluntary, with a study protocol almost identical to that in Study 1. E.g. all students, including those who did not consent to the study, were later provided with all the proofs in a single document to read and discuss at a subsequent course workshop.

In total, 172 students participated in judging. The median time taken for each judgement was 11 seconds. To ensure the results are not skewed by participants not taking the task seriously, we removed all judgements from judges whose median judgement time was under 5 seconds. This left 153 judges distributed across the judging sessions, as shown in Table 3. All judging sessions achieved an acceptable level of reliability as measured by the SSR, with the lowest being insight (0.724). However, the judging sessions for insight and understanding did not reach the commonly-used threshold of 0.7 for split-halves reliability (see Table 3). 


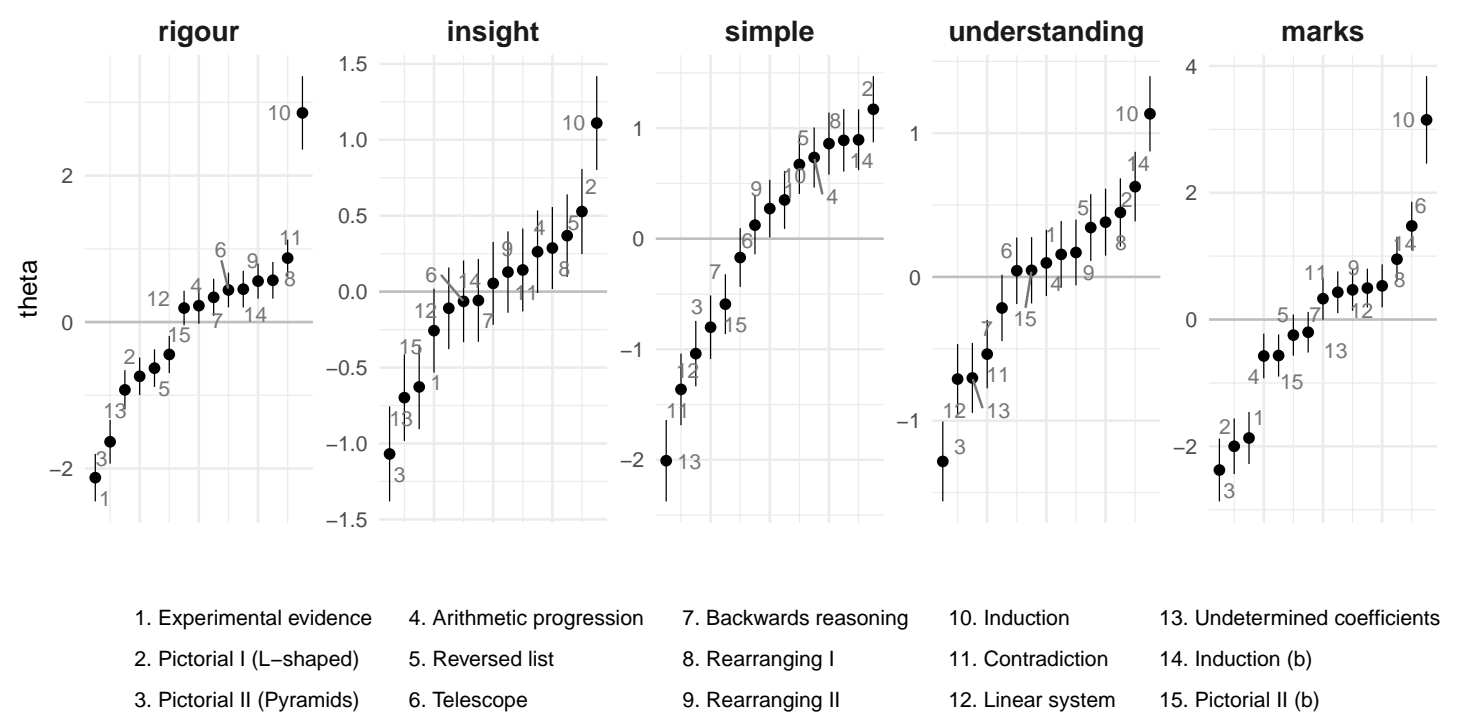

Figure 2: Scale scores (with standard error bars) produced by judges in Study 2, for each proof on each dimension. Note that scales are not directly comparable between conditions: see remarks below.

\subsection{Results}

The scale scores for the proofs on each dimension are shown in Figure 2. Correlations between the scores on the different dimensions are summarised in Figure 3, with full details in the Appendix (Table 6 and Figure 6). We will refer to these across the following sections, as we address each research question in turn.

\subsubsection{Replication of Study 1}

As in Study 1, both the rigour and insight judging groups produced scales with an acceptable level of reliability, as measured by SSR, although the reliability of the insight scale is lower-we return to this point in the next section.

The scales produced in Study 1 and Study 2 are very similar. For instance, on all scales 10 (induction) is top, while 1 (experimental evidence) and 3 (Pictorial II) are at the bottom. Overall, there is a strong correlation between the corresponding scales from the two studies, as shown in Figure 4. The correlation between the two scales for rigour is particularly strong, with a median estimated correlation coefficient of $r=0.91$.

In Study 1 we found a strong correlation between the rigour and insight scales. The same is true in Study 2: the median estimated correlation between the rigour and insight scales was $r=0.72$, with $B F=64.15$ representing strong evidence in favour of a correlation (see Table 6 in the Appendix).

\subsubsection{Investigating insight}

The results show that students have still found it difficult to distinguish proofs on the basis of our intended criterion of insight, whether using the adjusted wording that we introduced for insight or the new prompt for understanding. We will discuss each case in turn.

For the insight judging group, SSR was on the low end of acceptability, and split-halves reliability was very low-most likely because splitting the judging group in half brings the SSR for each half below the 


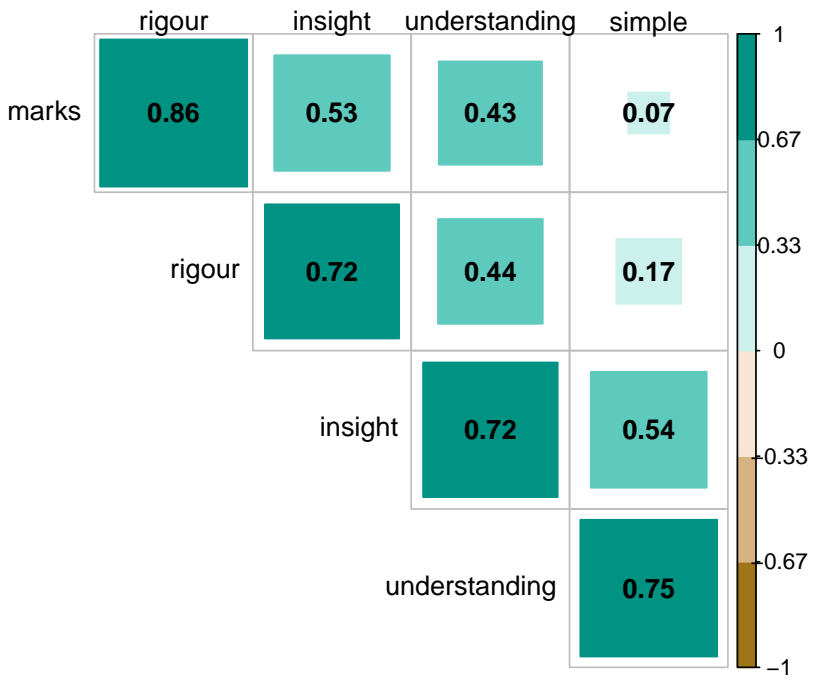

Figure 3: Summary of the median estimated correlation coefficient between the different scales in Study 2. Full details of the estimates are given in the Appendix, in Table 6.
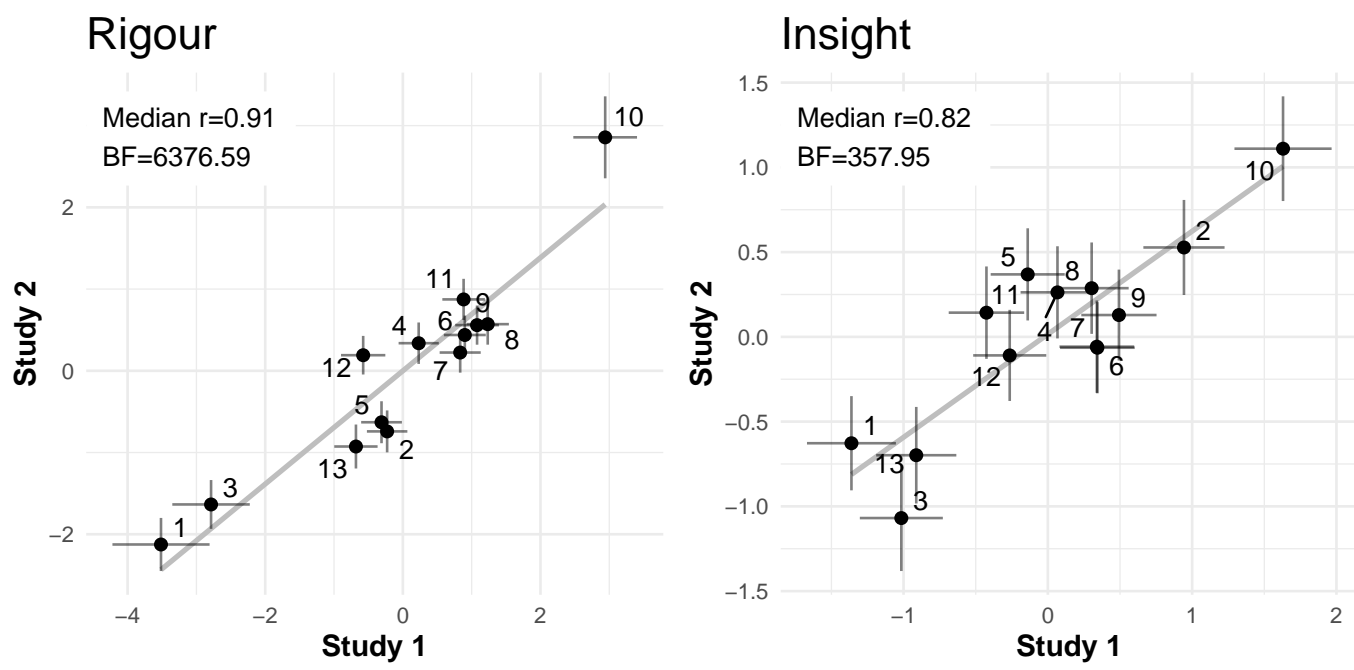

Figure 4: Correlation between the CJ scales for insight and rigour produced in Study 1 and Study 2. 
acceptable level. So, while the overall judging group has produced a marginally reliable scale for insight, it appears the judges were not in strong agreement about this criterion.

Lack of agreement between judges on the criterion was apparent in their responses to the prompt to explain what it means for a proof to give insight into why a theorem is true. Some students gave comments that align closely with the notions of insight identified by Weber (2010) and others.

... easy for the human mind to follow and thus build some degree of intuitiveness of the nature of the theorem, and why it is true. It can also connect mathematical concepts and ideas, thus giving insight into the structure of the subject being considered and mathematics in general.

However many students' comments were hard to distinguish from the responses given by students asked to explain what it means for a proof to be rigorous (e.g. with a focus on logical validity), even when using the word "insight" in their response.

The proof uses logical and deductive reasoning to unequivocally validify [sic] a theorem. ... Through this you can follow each step of reasoning, which have complete justification to gain an insight into why a theorem is true.

Judges in the understanding condition tended to show a similar preoccupation on the nature of proof as a rigorous argument, rather than how an individual might or might not understand the proof. However, as shown in Figure 3, while the understanding scale correlates strongly with insight $(r=0.72)$, it correlates only weakly with rigour $(r=0.44)$. Comparing the position of proofs on the understanding and rigour scales (Figure 2), there are some notable differences-contradiction (11) is near the bottom of the ranking for understanding, while it is second place on the rigour scale. And while experimental evidence (1) is at the bottom of the scale for rigour, it is in the middle of the understanding scale.

\subsubsection{Independence of judging criteria}

Since the rigour and insight scales were strongly correlated, we sought to rule out the possibility that judges were simply using some alternative criterion (e.g. "best") by identifying a criterion that would produce a dissimilar scale. The distinction between rigour and understanding described in the previous section has already provided some evidence of this. There are three further aspects of the results that help to establish the validity of the judging process.

First, we checked whether any of the five judging scales correlated with either word count or string length ${ }^{2}$. We found no evidence for this - the Bayes factors for the correlation tests ranged from 0.33 to 1.77, all representing weak evidence (see the supplementary materials for details). This gives confidence that the judges were not simply selecting the "longest" proof in each pair.

Second, we investigated the correlations of the simple scale with the others (see Figure 3). The wording of the simple condition was chosen with the expectation that it would not correlate with either rigour or insight. This was the case for simple-rigour: the correlation was $r=0.17$ with $B F=0.40$ representing weak evidence against the existence of a correlation, as is apparent in the scatterplot in Figure 5 (b). On the other hand, for simple-insight, there is moderate evidence in favour of a correlation $(B F=4.38)$, albeit a moderate one $(r=0.54)$.

The prompt for "simplicity" does appear to have invoked genuinely independent conceptions, which are a mixture of (i) length of the proof, and also (ii) ability to understand.

It should be able to be explained quite concisely/with few words, and the main idea of the proof should be able to be understood after reading it once.

\footnotetext{
${ }^{2}$ Since there is no universally agreed notion of word/symbol count the "length" of a proof is measured using both the number of English words, and the number of non-whitespace characters used in the representation of the proof by LTEX. These are shown in Table 5. For the pictorial proofs the reported length seriously under-estimates the "complexity" of the argument, but is reported anyway.
} 
(a)

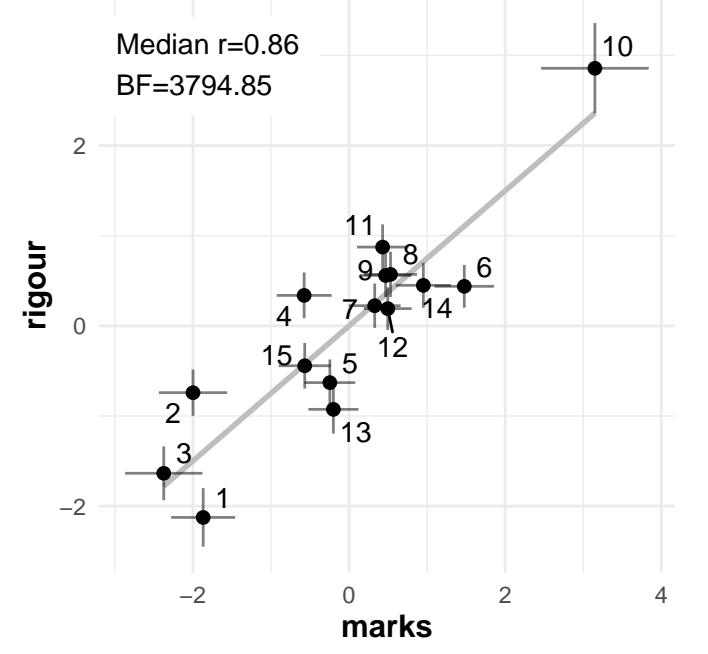

(b)

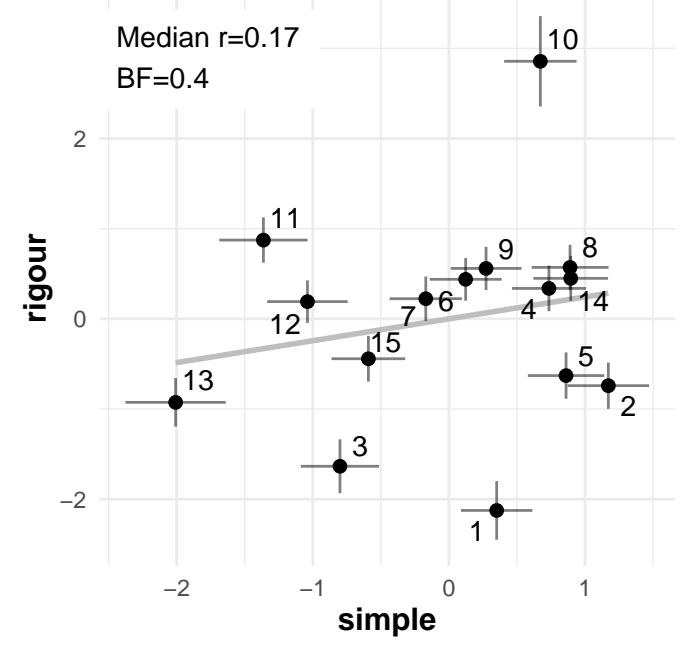

Figure 5: Correlation between scale scores for rigour and selected other dimensions in Study 2: (a) the highest correlation was observed between rigour and marks, (b) one of the lowest correlations was between rigour and simple, where $B F=0.4$ represents weak evidence against the existence of any correlation. Further correlations are shown in the Appendix, in Figure 6.

Indeed, it is notable that proof 2 (pictorial I) comes top on the simple scale while proof 10 (induction) is top of every other scale.

Third, the scale for marks had a wide range of correlations with other scales. On the one hand, there is moderate evidence against there being a correlation with simple $(B F=0.33)$, while on the other hand marks is strongly correlated with rigour $(r=0.86$, see Figure 5 (a)).

Finally, to better understand the relationship between the 5 dimensions, we carried out an exploratory factor analysis of the scores for the 15 proofs. This provides a way to determine if the dimension scores can be explained by a smaller number of factors. A similar approach was used by Inglis and Aberdein (2015) to form groups of related adjectives, where many participants had directly provided a score for each adjective. In our case, the scores come from CJ, and our "participants" are the 15 proofs. The Kaiser-Meyer-Olkin measure of sampling adequacy suggested that the data were suitable for factor analysis $(K M O=0.58)$, and Bartlett's test of sphericity confirmed that there is sufficient significant correlation in the data for factor analysis $\left(\chi^{2}(10)=63.23, p<.001\right)$. Parallel analysis supported extracting two factors, and we used a varimax rotation so that the extracted factors are orthogonal. The two latent factors account for $87.86 \%$ of the total variance of the original data $(F 1=46.02 \%, F 2=41.84 \%)$. Loadings of the dimensions onto the two factors are shown in Table 4, with high positive loadings suggesting the dimension is representative of that factor. The loadings show that Factor 1 is mainly associated with rigour and marks, while Factor 2 is mainly associated with simple and understanding. The insight dimension loads moderately onto both factors. This two-factor structure provides further demonstration that the various judging criteria led to independent (though in some cases, closely related) judgements.

\subsubsection{Modified proofs}

Proof by induction (10) was ranked most highly on all scales except simplicity (see Figure 2). The modified version, induction $b$ (14), that used a deliberately less formal style, was not considered as rigorous as induction (10). The two induction proofs are indistinguishable on simplicity, and both contribute high levels of understanding. Surprisingly perhaps, the lack of rigour in induction b (14) has not been seriously detrimental to the predicted marks: induction $\mathrm{b}$ (14) is ranked 3rd from the top for marks, with some 


\begin{tabular}{lll}
\hline Dimension & Factor 1 & Factor 2 \\
\hline rigour & $\mathbf{0 . 9 8 1}$ & 0.201 \\
marks & $\mathbf{0 . 8 8 9}$ & 0.112 \\
insight & $\mathbf{0 . 6 3 4}$ & $\mathbf{0 . 6 4 6}$ \\
understanding & 0.381 & $\mathbf{0 . 8 4 5}$ \\
simple & -0.008 & $\mathbf{0 . 9 5 3}$ \\
\hline
\end{tabular}

Table 4: Factor loadings for each of the dimensions in Study 2, on the two factors identified in the data. Strong loadings (with absolute value greater than 0.4 ) are shown in bold.

separation between it and the next 5 proofs (which are an indistinguishable group).

We included three pictorial proofs: 2 (pctorial I), 3 (pictorial II) and 15 (pictorial IIb). Proof 2 (pictorial I), perhaps showed the most variety of any proof in its position in the rankings, appearing very low on rigour and marks, but very high on simplicity, insight and understanding. In contrast, 3 (pictorial II) appears to be the consistently lowest ranked proof for all but simplicity. Yet, adding some modest words of explanation to create 15 (pictorial IIb) provided significantly more rigour, understanding and marks.

\subsection{Discussion}

We found that participants were able to distinguish proofs on the five subjective dimensions. However, as in Study 1, the scores for rigour and insight were highly correlated — even in spite of our efforts to strengthen the prompt for insight. The results from other dimensions (understanding, simple and marks) show that there were genuinely independent judgements being made on the different dimensions, as confirmed by low correlations between certain dimensions (e.g. rigour and simple). While some of the dimensions are closely related, an exploratory factor analysis found that the dimensions can be explained by two underlying factors—one associated with rigour/marks, and another with simple/understanding.

In terms of our overarching research focus, on whether rigour and insight can be distinguished as separate constructs, these results add to those of Study 1 in suggesting that they cannot-at least by this group of participants and using our methodology. We will return to possible explanations for this in section 5, but for now we reiterate that there is some evidence from the comments left by students that they did not understand the wording of our prompts ("which proof gives more insight into why the theorem is true?") in the intended way.

In this study, we were also interested in whether any modifications of certain proofs would affect their scores in expected ways. We found that reducing the formality of the proof by induction did indeed substantially reduce its perceived rigour, despite the changes being relatively minor (see proofs 10 and 14 in Appendix A). This suggests that in future work using similar methodology, careful attention should be paid to the presentation of the proofs, particularly when drawing conclusions. For instance, our findings here show that a proof by induction is not viewed as inherently rigorous if details are omitted in the presentation. Similarly, we included three picture proofs in this study and found that they received a range of subjective judgements. As expected, adding text to a picture proof increased its perceived rigour.

\subsubsection{Features of individual proofs}

In this section we make some observations about individual proofs.

Why is induction ranked so highly on all scales? We are not surprised that induction is both (i) rigorous and (ii) is worthy of high marks, but that induction clearly gives most insight and understanding into why a theorem is true is surprising. Even on "simplicity" both forms of induction (10/14) were ranked positively, and relatively highly on the scale (14 is second to 2). Induction is a rare example of proof being taught in a specific, structured way (Alarfaj \& Sangwin, 2020), so it may be that students' familiarity with this style 
of proof is shaping their judgements.

In Study 1, we deferred a discussion of the problem solving proofs, i.e. those which find the sum of the series in the course of the proof, rather than assuming it a priori. Our hypothesis was that problem solving proofs would be judged to provide more "insight" than proofs which rely on a prioi knowledge of the result. Proof 8 (rearranging I) is problem-solving and also arguably has the widest range of other situations to which the proof technique can readily be applied and was judged favorably on all scales, but is not clearly distinguished on any scale. Two other proofs which readily provide the sum of a series in a wide range of situations are 12 (linear systems) and 13 (undetermined coefficients). We looked for, but could see no patterns whatsoever between problem solving proofs. We predicted such characteristics would be relevant in the rankings. However, on reflection we do not routinely use multiple proofs of the same result or discuss such aesthetic considerations with our students and so it is unlikely they are attuned to considering such characteristics when making judgements. Furthermore, it is hard to know which proofs (subsequently) generalise to other situations as a year 1 undergraduate student.

Undetermined coefficients (13) has not been popular with this group. This is a group of year 1 undergraduate students. Undetermined coefficients relies, to some extent, with familiarity with dealing with infinite series and this topic is on the boundary of their mathematical experience, especially using series in this kind of informal way. Lack of familiarity may have contributed to the rankings here. Is 13 perceived to be so low on rigour because of the infinite series "hand-waiving" step, and what modifications to the proof could we make which would raise the judged level of rigour?

\section{General discussion}

Across two studies, we found that undergraduate students could distinguish between proofs of a particular theorem on a range of subjective dimensions. Some of these dimensions appear to be distinct, e.g. rigour and simplicity, as evidenced by an absence of correlation between the scores assigned to proofs on the two dimensions. However, for the main distinction we were interested in - rigour versus insight—our participants made very similar judgements about the proofs. We suggest three possible reasons for this.

First, as already noted, participants' written comments showed that many of them did not interpret our prompts ("which proofs gives more insight?"/"which proof gives more insight into why the theorem is true?") in the intended way. This likely explains the relatively low reliability attained for the insight scale in both studies, as the judges will have been using a mix of criteria to make their judgements. On the one hand, it may be that our participants do have an understanding of what makes a proof insightful (in the sense often referred to by mathematics educators, as outlined in section 1) and further work would be needed to devise a prompt that suitably triggers this. On the other hand, it may be that many undergraduate students in this group (and perhaps more generally) do not appreciate this subjective dimension-we return to the educational implications of this possibility in section 6.

Second, the low reliability and unexpected results for the insight scale may be signs of a methodological issue. It could be that comparative judgement is the wrong tool to reliably measure insight, which is often described as arising in a sudden, "magical" moment (Barnes, 2000). When judges are making a series of paired comparisons, their sense of insight from subsequent proofs might be altered. Their sense could be dulled, e.g. through boredom, or heightened, e.g. making a novel connection once a basic result is accepted. Inglis and Mejía-Ramos (2019) suggest that, in making (epistemic) judgements about the explanatoriness of proofs, "we are imagining a hypothetical audience and judging the extent to which the proof will generate understanding for them." In our case, the judges are presumably thinking about a hypothetical person seeing a given pair of proofs for the first time. Even if judges are able to do this reliably, a possible weakness of the CJ approach is that it does not account for how later judgements may be influenced by repeated exposure during earlier judgements. Further work should investigate this issue, perhaps by systematically controlling the order in which certain proofs are first shown to judges.

Third, our results are based on a single set of proofs of a single theorem. It could be that with a different collection of proofs, participants would have been better able to distinguish insight and rigour. However, 
as discussed previously, participants' responses to the definition prompts suggest they simply did not distinguish insight and rigour even before seeing the proofs. Still, further work to replicate our findings using a different theorem and proofs would be welcome.

Our results may also have been affected by the novelty of the activity for our participants. It is likely that the students participating in these studies have never been asked to compare a wide variety of proofs of the same theorem, and that they are not accustomed to making aesthetic judgements or value judgements about proofs (such as choosing between two proofs of the same theorem). We do not think this represents a serious threat to our results, as the high reliability achieved in most judging sessions suggests that students were nevertheless capable of making these judgements.

\section{Conclusion}

The main contribution of this paper is to establish that the comparative judgement method can be used to measure and compare subjective judgements of proofs on different dimensions. In particular, we found that rigour is a reliable scale. The fact that the undergraduate students in our studies did not distinguish between rigour and insight, and did not appear to appreciate the commonly-used description of "insight into why a theorem is true", has clear educational implications.

The importance placed on insight by mathematics educators (e.g., Hanna, 1990; Hersh, 1993; Smith, 2006; Stylianides et al., 2016) seems to be at odds with the aesthetic sense being developed by undergraduate students in our studies. Notably, we found that the distinction between insight and rigour was not apparent to either first-year undergraduates (in Study 2) or third-year undergraduates (in Study 1), giving some indication that students are not developing this aesthetic sense over time.

It will be important to determine if there is a difference in behaviour between undergraduate students and expert mathematicians. Such differences have already been noted in other aspects of behaviour with regard to proofs. For instance, when reading proofs to validate them, "compared to mathematicians, undergraduate students spend proportionately more time fixating on formulae" (Inglis \& Alcock, 2012, p. 379), and "undergraduate students tend to be more sceptical of the reliability of visual arguments than their teachers" (Inglis \& Mejía-Ramos, 2009, p. 107). So, it would not be surprising if expert mathematicians have an appreciation of "insight" that few undergraduate students share.

If it is possible for expert mathematicians to distinguish proofs on the basis of insight, it would be interesting to see if an intervention could help students to better understand the notion. This could be as simple as a more extensive briefing before the comparative judgement exercise, e.g. presenting some examples of proofs with an accompanying narrative explaining how expert mathematicians appraise them in terms of their insight. Alternatively, it could be based on a longer-term intervention throughout a course on proof (see, e.g., Miller \& CadwalladerOlsker, 2020). Developing students' aesthetic sense in this way would be consistent with the view that "proof instruction must also seek to expose the underlying values that guide that practice. It is insufficient to train students in the norms of proof if students never develop some means of understanding and justifying their purpose." (Dawkins \& Weber, 2017, p. 138).

If it is not possible for expert mathematicians to distinguish proofs on the basis of insight then it would seriously call into question the existence of "magical" moments as a scientifically meaningful construct, however ubiquitously individuals report personal experiences of such moments. If insight is a consciousbut fleeting - discontinuity in understanding for an individual, then it cannot be described as an intrinsic property of a proof and would be impossible to measure using comparative judgement. This possibility also has educational implications. Our experiments suggest we can establish particular proofs are reliably judged to have characteristics such as rigour and simplicity. Rigour was related to marks which is an extrinsic value judgement, and simplicity was related to developing personal understanding. Can teachers design particular proofs which engineer situations that reliably provide moments of insight? Careful assessment design might engineer moments of insight, but we found no evidence for this in our experiments. 


\section{Declarations}

Funding: this research was funded by the University of Edinburgh. Ethics approval was granted by the University of Edinburgh. Consent to participate was sought and freely given, and participation in this study was voluntary. We are aware of no conflicts of interest.

Data was gathered using the Moodle "Assignment" module, with the optional comparative judgement submission plugin. This code is open-source and freely available. The data and analysis code are openly available at

http://doi.org/10.5281/zenodo. 4893915

\section{Acknowledgements}

Thanks to Dr Ian Jones of Loughborough University for overseeing the development of the CJ software used in this study. Thanks to Professor Matthew Inglis for helpful discussion on background to this study.

\section{References}

Aigner, M., \& Ziegler, G. (2018). Proofs from THE BOOK (Sixth). Springer.

Alarfaj, M., \& Sangwin, C. J. (2020). Mathematical induction in advanced higher mathematics. Scottish Mathematics Council Journal, 50, 79-86.

Avigad, J. (2018). Opinion: The mechanization of mathematics. Notices of the American Mathematical Society, 65(6), 681-690. https://doi.org/10.1090/noti1688

Barnes, M. (2000). 'magical' moments in mathematics: Insights into the process of coming to know. For the Learning of Mathematics, 20(1), 33-43. http://www.jstor.org/stable/40248316

Bell, A. W. (1976). A study of pupils' proof-explanations in mathematical situations. Educational Studies in Mathematics, 7(1), 23-40. https://doi.org/10.1007/BF00144356

Bisson, M. J., Gilmore, C., Inglis, M., \& Jones, I. (2016). Measuring conceptual understanding using comparative judgement. International Journal of Research in Undergraduate Mathematics Education, 2(2), 141-164. https://doi.org/10.1007/s40753-016-0024-3

Bramley, T. (2015). Investigating the reliability of adaptive comparative judgment (Cambridge Assessment Research Report). Cambridge Assessment. Cambridge, UK.

Brown, J. R. (2008). Philosophy of mathematics: A contemporary introduction to the world of proofs and pictures. Routledge. https://doi.org/10.4324/9780203932964

Burn, R. P. (2010). Sums of powers of integers - how Fermat may have found them. The Mathematical Gazette, 94(529), 18-26. https://doi.org/10.2307/27821884

Clark, H. H. (1973). The language-as-fixed-effect fallacy: A critique of language statistics in psychological research. Journal of Verbal Learning and Verbal Behavior, 12(4), 335-359. https://doi.org/10. 1016/S0022-5371(73)80014-3

Davies, B., Alcock, L., \& Jones, I. (2020). Comparative judgement, proof summaries and proof comprehension. Educational Studies in Mathematics, 105(2), 181-197. https://doi.org/10.1007/s10649020-09984-x

Davies, B., Alcock, L., \& Jones, I. (2021). What do mathematicians mean by proof? A comparativejudgement study of students' and mathematicians' views. The Journal of Mathematical Behavior, 61, 100824. https://doi.org/10.1016/j.jmathb.2020.100824

Dawkins, P. C., \& Weber, K. (2017). Values and norms of proof for mathematicians and students. Educational Studies in Mathmatics, 95(2), 129-142. https://doi.org/10.1007/s10649-016-9740-5

Dedekind, R. (1963). Essays on the theory of numbers. Dover.

D’Ooge, M. L. (1926). Nicomachus of Gerasa: Introduction to arithmetic. MacMillan.

Edwards, A. W. F. (1982). Sums of powers of integers: A little of the history. The Mathematical Gazette, 66(435), 22-28. https://doi.org/10.2307/3617302 
Euler, L. (1822). Elements of algebra (3rd) [Translated from the French, with the notes of M. Bernoulli and the Additions of M. de La Grange, by Hewlett, J.]. Longman, Hurst, Rees, Orme; Co.

Freudenthal, H. (1981). Major problems of mathematics education. Educational Studies in Mathematics, 12(3), 133-150.

Hamami, Y., \& Morris, R. L. (2020). Philosophy of mathematical practice: A primer for mathematics educators. ZDM - Mathematics Education, 52(6), 1113-1126. https://doi.org/10.1007/s11858020-01159-5

Hanna, G. (1990). Some pedagogical aspects of proof. Interchange, 21(1), 1573-1790. https://doi.org/10. 1007/BF01809605

Hanna, G., \& Mason, J. (2016). Key ideas and memorability in proof. For the Learning of Mathematics, $34(2), 12-16$.

Harel, G. (2014). Vital direction for mathematics education research.

Healy, L., \& Hoyles, C. (2000). A study of proof conceptions in algebra. Journal for Research in Mathematics Education, 31(4), 396-428.

Hersh, R. (1993). Proving is convincing and explaining. Educational Studies in Mathematics, 24, 389-399. https://doi.org/10.1007/BF01273372

Inglis, M., \& Aberdein, A. (2015). Beauty is not simplicity: An analysis of mathematicians' proof appraisals. Philosophia Mathematica, 23(1), 87-109. https://doi.org/10.1093/philmat/nku014

Inglis, M., \& Aberdein, A. (2016). Diversity in proof appraisal. In Mathematical cultures (pp. 163-179). Springer International Publishing.

Inglis, M., \& Alcock, L. (2012). Expert and novice approaches to reading mathematical proofs. Journal for Research in Mathematics Education, 43, 358-390.

Inglis, M., \& Mejía-Ramos, J. P. (2009). On the persuasiveness of visual arguments in mathematics. Foundations of Science, 14(1-2), 97-110. https://doi.org/10.1007/s10699-008-9149-4

Inglis, M., \& Mejía-Ramos, J. P. (2019). Functional explanation in mathematics. Synthese, 1-24. https: //doi.org/10.1007/s11229-019-02234-5

Jones, I., \& Alcock, L. (2014). Peer assessment without assessment criteria. Studies in Higher Education, 39(10), 1774-1787. https://doi.org/10.1080/03075079.2013.821974

Jones, I., Bisson, M., Gilmore, C., \& Inglis, M. (2019). Measuring conceptual understanding in randomised controlled trials: Can comparative judgement help? British Educational Research Journal, 45(3), 662-680. https://doi.org/10.1002/berj.3519

Jones, I., Swan, M., \& Pollitt, A. (2014). Assessing mathematical problem solving using comparative judgement. International Journal of Science and Mathematics Education, 13(1), 151-177.

Jones, I. (2021). Comparative Judgement plugin for the Moodle Assignment, https://github.com/ianjones/ moodle-assignsubmission_comparativejudgement.

Kalman, D. (1993). Six ways to sum a series. The College Mathematics Journal, 24(5), 402-421.

Loomis, E. (1968). The Pythagorean proposition. National Council of Teachers of Mathematics.

MacKenzie, D. (2004). Mechanizing proof: Computing risk and trust. The MIT Press, Cambridge, Massachusetts.

Mejía-Ramos, J. P., Evans, T., Rittberg, C., \& Inglis, M. (2021). Mathematicians' assessments of the explanatory value of proofs. Axiomathes, 1-25. https://doi.org/10.1007/s10516-021-09545-8

Miller, D., \& CadwalladerOlsker, T. (2020). Investigating undergraduate students' view of and consistency in choosing empirical and deductive arguments. Research in Mathematics Education, 22(3), 249264. https://doi.org/10.1080/14794802.2019.1677489

Nelson, R. B. (1993). Proofs without words: Exercises in visual thinking. The Mathematical Association of America.

Ording, P. (2019). 99 variations on a proof. Princeton University Press.

Pollitt, A. (2012). The method of adaptive comparative judgement. Assessment in Education: Principles, Policy \& Practice, 19(3), 281-300. https://doi.org/10.1080/0969594X.2012.665354

Polya, G. (1962). Mathematical discovery: On understanding, learning, and teaching problem solving. Wiley.

Raman-Sundström, M., \& Öhman, L. (2016). Mathematical fit: A case study. Philosophia Mathematica, 26(2), 184-210. https://doi.org/10.1093/philmat/nkw015

Sangwin, C. J. (2023). Sums of the first odd integers. The Mathematical Gazette). 
Sangwin, C. J., \& Bickerton, R. (2021). Practical online assessment of mathematical proof. International Journal of Mathematical Education in Science and Technology. https : // doi . org / 10 . 1080 / 0020739X.2021.1896813

Smith, J. C. (2006). A sense-making approach to proof: Strategies of students in traditional and problembased number theory courses. Journal of Mathematical Behavior, 25(1), 73-90. https://doi.org/ 10.1016/j.jmathb.2005.11.005

Stylianides, G. J., Sandefur, J., \& Watson, A. (2016). Conditions for proving by mathematical induction to be explanatory. The Journal of Mathematical Behavior, 43, 20-34. https://doi.org/10.1016/j. jmathb.2016.04.002

Thurstone, L. L. (1927). A law of comparative judgment. Psychological Review, 34(4), 273-286.

Todhunter, I. (1897). Algebra for the use of colleges and schools (5th). MacMillian; Co.

Tuckey, C. O., \& Armistead, W. (1962). Algebra (3rd). Longmans, Green \& Co.

van Doorn, J., van den Bergh, D., Böhm, U., Dablander, F., Derks, K., Draws, T., Etz, A., Evans, N. J., Gronau, Q. F., Haaf, J. M., Hinne, M., Kucharský, Š., Ly, A., Marsman, M., Matzke, D., Gupta, A. R. K. N., Sarafoglou, A., Stefan, A., Voelkel, J. G., \& Wagenmakers, E. J. (2020). The JASP guidelines for conducting and reporting a Bayesian analysis. https://doi.org/10.3758/s13423-02001798-5

Verhavert, S., De Maeyer, S., Donche, V., \& Coertjens, L. (2018). Scale separation reliability: What does it mean in the context of comparative judgment? Applied Psychological Measurement, 42(6), 428445. https://doi.org/10.1177/0146621617748321

Weber, K. (2010). Proofs that develop insight. For the Learning of Mathematics, 30(1), 32-36. 


\section{A The proofs}

Students were not shown the name of the proof or provenance (where known) during the experiment.

\section{Example 1 Experimental evidence}

$$
\begin{gathered}
1=1=1^{2} \\
1+3=4=2^{2} \\
1+3+5=9=3^{2} \\
1+3+5+7=16=4^{2} \\
1+3+5+7+9=25=5^{2} \\
1+3+5+7+9+11=36=6^{2} \\
\vdots \\
1+3+5+7+\cdots+(2 n-1)=n^{2} .
\end{gathered}
$$

\begin{tabular}{|c|c|c|c|c|c|c|c|c|}
\hline 웅 & 0 & (ㄱ) & 8 & & 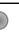 & (9) & (ㅇ) & 3 \\
\hline 0 & 0 & 0 & 8 & & 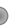 & 0 & 0 & C \\
\hline 0 & 0 & 0 & $C$ & & D & ○ & 0 & C \\
\hline 0 & 0 & 0 & $\mathrm{C}$ & & 7 & ? & 0 & (6) \\
\hline 0 & 0 & 0 & $c$ & & 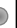 & 0 & 0 & C \\
\hline 0 & 0 & O & ( & & 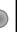 & 0 & 0 & C \\
\hline 0 & 0 & 10 & 0 & & 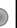 & ○ & 0 & C \\
\hline 0 & 0 & 0 & & & 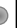 & ; & 0 & $c$ \\
\hline
\end{tabular}

Example 2 Pictorial I Nicomachus of Gerasa, circa 100CE: (D'Ooge, 1926, pg. 243), (Nelson, 1993, pg. 71).

$$
1+3+5+7+\cdots+(2 n-1)=n^{2} .
$$

Example 3 Pictorial II (Nelson, 1993, pg. 72)

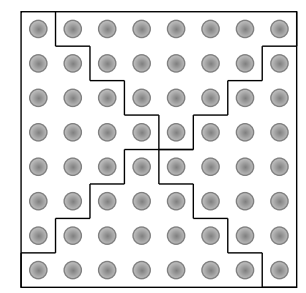

$$
1+3+5+7+\cdots+(2 n-1)=\frac{1}{4}(2 n)^{2}=n^{2} .
$$

Example 4 Arithmetic Progression (Euler, 1822, §422)

$$
1+3+5+7+\cdots+(2 n-1)
$$

is an arithmetic progression with difference 2 and $n$ terms. The first term $a_{1}=1$, and the last term $a_{n}=$ $2 n-1$, and the sum of an AP is $\frac{n}{2}\left(a_{1}+a_{n}\right)$, which in this case is $\frac{n}{2}(1+2 n-1)=n^{2}$. 
Example 5 Reversed list Write the terms twice, with the second list reversed.

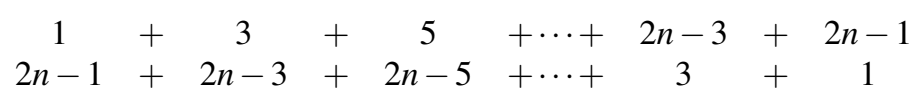

Each column has total $2 n$ and there are $n$ columns. So the total is $2 n^{2}$ proving $\sum_{k=1}^{n}(2 k-1)=n^{2}$.

\section{Example 6 Telescope}

Notice that $2 k-1=k^{2}-(k-1)^{2}$, so that adding up we have

$$
\sum_{k=1}^{n}(2 k-1)=\sum_{k=1}^{n} k^{2}-(k-1)^{2} .
$$

However in

$$
\sum_{k=1}^{n} k^{2}-(k-1)^{2}=\left(1^{2}-0^{2}\right)+\left(2^{2}-1^{2}\right)+\left(3^{2}-2^{2}\right)+\cdots+\left(n^{2}-(n-1)^{2}\right)
$$

all terms cancel except two, one from the first term and one from the last, i.e. $-0^{2}+n^{2}$, leaving $n^{2}$.

Example 7 Backwards reasoning (Tuckey \& Armistead, 1962, pg. 210)

The Fundamental Theorem of Finite Differences says that $S_{n}=\sum_{k=1}^{n} a_{k}$ if and only if $a_{n}=S_{n}-S_{n-1}$.

Consider $S_{n}=n^{2}$ then $S_{n}-S_{n-1}=n^{2}-(n-1)^{2}=2 n-1$. Hence $\sum_{k=1}^{n}(2 k-1)=n^{2}$.

\section{Example 8 Rearranging I}

We use the standard results $\sum_{k=1}^{n} k=\frac{n(n+1)}{2}$ and $\sum_{k=1}^{n} 1=n$ and rearrange

$$
\sum_{k=1}^{n}(2 k-1)=2 \sum_{k=1}^{n} k-\sum_{k=1}^{n} 1=2 \frac{n(n+1)}{2}-n=n^{2} .
$$

\section{Example 9 Rearranging II}

We use the standard result $\sum_{k=1}^{n} k=\frac{n(n+1)}{2}$ and rearrange

$$
\begin{gathered}
\sum_{k=1}^{n} \underbrace{2 k-1}_{\text {odd }}=(\underbrace{1+2+3+\cdots+2 n}_{\text {all }})-(\underbrace{2+4+6+\cdots+2 n}_{\text {even }}) \\
=(1+2+3+\cdots+2 n)-2(1+2+3+\cdots+n) \\
\\
\sum_{k=1}^{n}(2 k-1)=\sum_{k=1}^{2 n} k-2 \sum_{k=1}^{n} k \\
=\frac{2 n(2 n+1)}{2}-2 \frac{n(n+1)}{2}=n^{2} .
\end{gathered}
$$

\section{Example 10 Induction}

Let $P(n)$ be the statement $\sum_{k=1}^{n}(2 k-1)=n^{2}$.

Since $\sum_{k=1}^{1}(2 k-1)=1=1^{2}$ we see $P(1)$ is true.

Assume $P(n)$ is true then

$$
\sum_{k=1}^{n+1}(2 k-1)=\sum_{k=1}^{n}(2 k-1)+(2(n+1)-1)=n^{2}+2 n+1=(n+1)^{2} .
$$


Hence $P(n+1)$ is true.

Since $P(1)$ is true and $P(n+1)$ follows from $P(n)$ we conclude that $P(n)$ is true for all $n$ by the principle of mathematical induction.

\section{Example 11 Contradiction}

To prove $\forall n \in \mathbb{N}: \sum_{k=1}^{n}(2 k-1)=n^{2}$, assume, for a contradiction, that $\exists n \in \mathbb{N}: \sum_{k=1}^{n}(2 k-1) \neq n^{2}$. Let $n^{*}$ be the smallest such example. Note, $n^{*}>1$ since $(2 \times 1)-1=1^{2}$.

If $\sum_{k=1}^{n^{*}}(2 k-1)>n^{* 2}$ then

$$
\sum_{k=1}^{n^{*}}(2 k-1)=2 n^{*}-1+\sum_{k=1}^{n^{*}-1}(2 k-1)>n^{* 2}
$$

and so

$$
\sum_{k=1}^{n^{*}-1}(2 k-1)>n^{* 2}-2 n^{*}+1=\left(n^{*}-1\right)^{2}
$$

This proves $\sum_{k=1}^{n^{*}-1}(2 k-1) \neq\left(n^{*}-1\right)^{2}$, which contradicts the minimality of $n^{*}$. The case $\sum_{k=1}^{n^{*}}(2 k-1)<n^{* 2}$ leads to an identical contradiction.

\section{Example 12 Linear system}

Since the sum is always an integer and $S_{n}=\sum_{k=1}^{n}(2 k-1) \leq n(2 n-1)$ the growth of $S_{n}$ is quadratic in $n$. We therefore assume

$$
\sum_{k=1}^{n}(2 k-1)=a n^{2}+b n+c \quad \forall n \in \mathbb{N} .
$$

Since this formula holds for all $n$ it must hold for $n=1,2,3$. Hence

$$
\begin{array}{lll}
1 & =a+b+c, & (n=1) \\
1+3 & =4 a+2 b+c, & (n=2) \\
1+3+5 & =9 a+3 b+c, & (n=3)
\end{array}
$$

This is a linear system in $a, b, c$ which we set up as

$$
\left(\begin{array}{lll}
1 & 1 & 1 \\
4 & 2 & 1 \\
9 & 3 & 1
\end{array}\right)\left(\begin{array}{l}
a \\
b \\
c
\end{array}\right)=\left(\begin{array}{l}
1 \\
4 \\
9
\end{array}\right) .
$$

The matrix clearly has non-zero determinant, so the system has a unique solution. This solution is (exercise to check) $a=1, b=c=0$. Hence $\sum_{k=1}^{n}(2 k-1)=n^{2}$.

Note, during this study this proof contained an unintended typographical mistake, with $2 b$ and $3 b$ mistakenly appearing as $2 n$ and $3 n$. Since none of the students in study 2 , rigour group, commented on this mistake we infer that it was not a significant detriment to this experiment.

Example 13 Undetermined coefficients (Todhunter, 1897, pg. 408)

Assume $1+3+5+7+\cdots+(2 n-1)=A+B n+C n^{2}+D n^{3}+E n^{4}+\cdots$. Then

$1+3+5+7+\cdots+(2 n-1)+(2(n+1)-1)=A+B(n+1)+C(n+1)^{2}+D(n+1)^{3}+E(n+1)^{4}+\cdots$

Subtracting

$$
2 n+1=B+C(2 n+1)+D\left(3 n^{2}+3 n+1\right)+E\left(4 n^{3}+6 n^{2}+4 n+1\right)+\cdots
$$

Equating powers of $n^{2}, n^{3}, \cdots$ on both sides we see $D=E=\ldots=0$.

$$
2 n+1=B+C(2 n+1) .
$$

Hence $B=0$ and $C=1$, from which $A=0$ and so $\sum_{k=1}^{n}(2 k-1)=n^{2}$. 


\section{Example 14 Induction (b)}

Consider the conjecture $\sum_{k=1}^{n}(2 k-1)=n^{2}$. First note $\sum_{k=1}^{1}(2 k-1)=1=1^{2}$. Now,

$$
\sum_{k=1}^{n+1}(2 k-1)=\sum_{k=1}^{n}(2 k-1)+(2(n+1)-1)=n^{2}+2 n+1=(n+1)^{2} .
$$

Hence $\sum_{k=1}^{n}(2 k-1)=n^{2}$ by induction.

\section{Example 15 Pictorial II (b)}

In the picture below, the stepped triangle at the bottom has $1+3+5+\cdots+(2 n-1)$ dots.

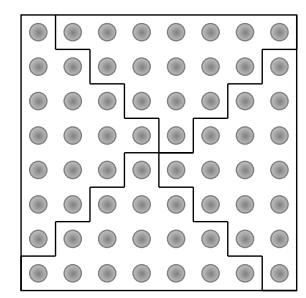

Four copies of this triangle can be fitted together to give a square where the side length is $(2 n-1)+1$ so the number of dots is $(2 n)^{2}$. Hence

$$
1+3+5+7+\cdots+(2 n-1)=\frac{1}{4}(2 n)^{2}=n^{2} .
$$

\section{B Participant information}

\section{B.1 Participant information for study 1}

To prepare for workshop 2 we are asking you to read through a number of proofs of the same theorem.

Theorem: The sum of the first $n$ odd integers, starting from 1 , is $n^{2}$.

Expressed in algebraic notation, this theorem becomes

$$
1+3+5+7+\cdots+(2 n-1)=\sum_{k=1}^{n}(2 k-1)=n^{2} .
$$

In order to create some data to discuss during the workshop we would like you to take part in a "comparative judgement" task well before the workshop itself. This involves reading two proofs at a time and choosing the "best" proof, with an optional justification. We will then rank order the proofs according to the preferences stated by the group.

We would also, potentially, like to publish a research paper based on this data and so at the end of this quiz we seek your formal consent to include anonymised responses in a research study. Whether you take part in the study or not is up to you: you still need to read the proofs to prepare for workshop 2.

This "quiz" and the comparative judgement assignment (CJ) are not assessed parts of this course, but they will help us to have a well-informed discussion during Workshop 2.

\section{Results}




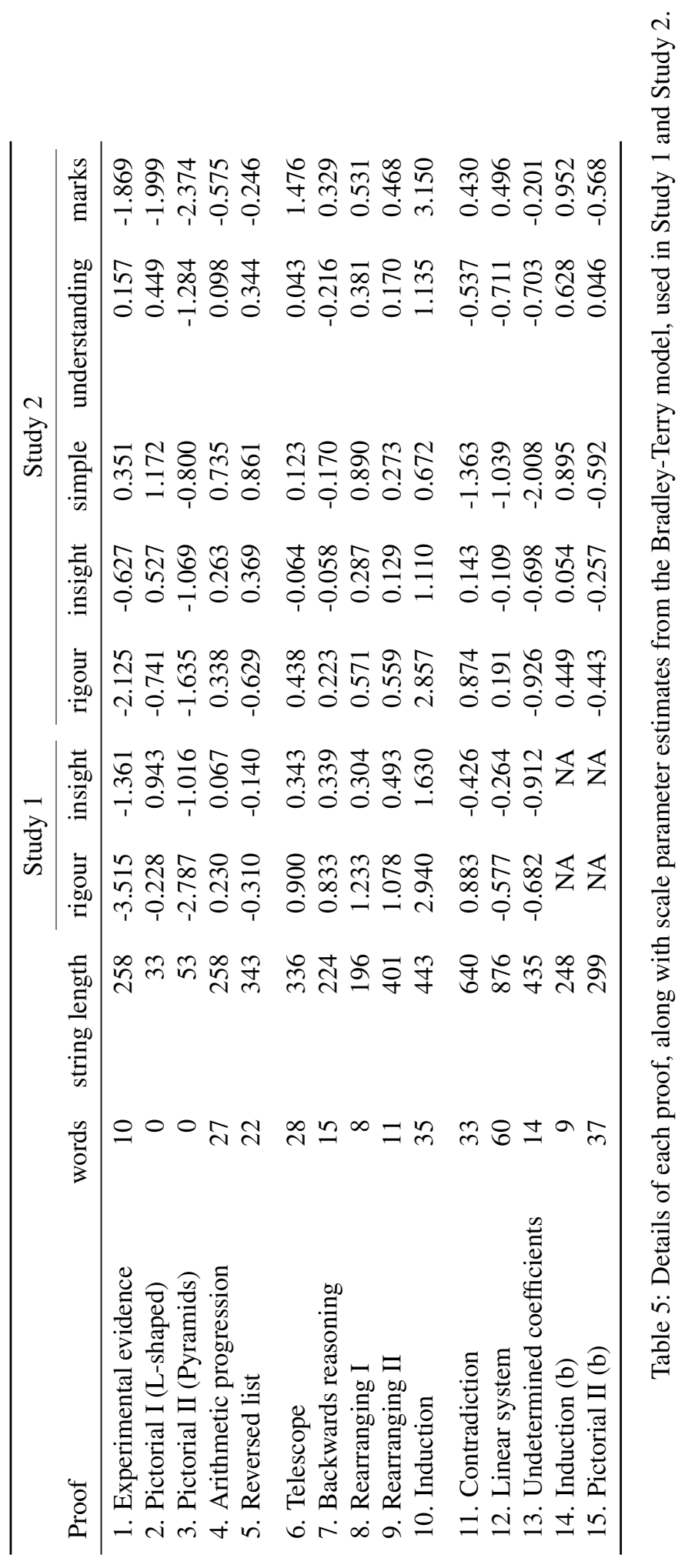




\begin{tabular}{llrlrl}
\hline Dimension 1 & Dimension 2 & Median & $95 \%$ HDI & BF & Interpretation \\
\hline marks & rigour & 0.86 & {$[0.66,0.96]$} & 3794.85 & Strong in favour \\
understanding & simple & 0.75 & {$[0.43,0.93]$} & 118.09 & \\
understanding & insight & 0.72 & {$[0.42,0.94]$} & 83.81 & \\
insight & rigour & 0.72 & {$[0.4,0.9]$} & 64.15 & \\
simple & insight & 0.54 & {$[0.1,0.83]$} & 4.38 & Moderate in favour \\
marks & insight & 0.53 & {$[0.1,0.84]$} & 4.35 & \\
understanding & rigour & 0.44 & {$[-0.01,0.77]$} & 1.75 & Weak in favour \\
marks & understanding & 0.43 & {$[-0.04,0.76]$} & 1.36 & \\
simple & rigour & 0.17 & {$[-0.33,0.58]$} & 0.40 & Weak against \\
marks & simple & 0.07 & {$[-0.41,0.52]$} & 0.33 & Moderate against \\
\hline
\end{tabular}

Table 6: Correlations between the dimensions in Study 2, given as the median and 95\% HDI for the posterior estimate of the correlation coefficient. Also shown are the Bayes factors for the strength of evidence supporting the correlation relative to the null model of no correlation, along with the standard interpretation of these according to van Doorn et al. (2020). 


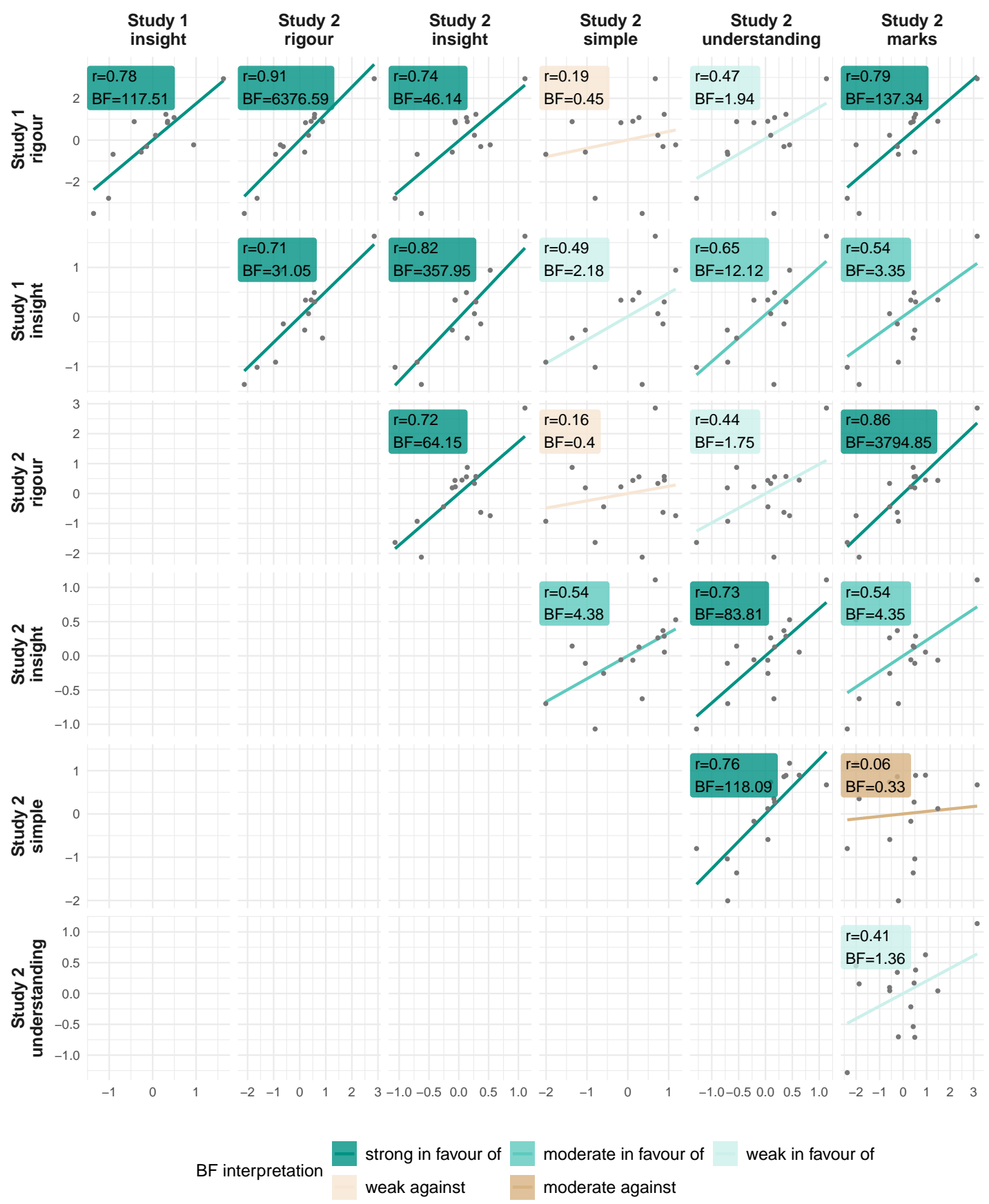

Figure 6: Visual summary of all correlations between scales produced by judges in Study 1 and Study 2 . Each point represents the position of one of the proofs on the two dimensions. The labels in the top left of each panel indicate the median estimate for the correlation coefficient (Pearson's $r$ ) along with the Bayes factor indicating the strength of evidence in favour of the existence of a correlation. Shading indicates the interpretation of the Bayes factors according to van Doorn et al. (2020). 